\title{
Risk and childbirth in psychiatry
}

\section{Margaret Oates}

The relationship between childbirth and serious mental illness has been known since the time of Ancient Greece. It was first described in the psychiatric literature by Esquirol and later by his pupil Marcé in 1857. A substantial number of women become mentally ill, often for the first time, following childbirth. There are few events associated with such a measurable and predictable risk to mental health as childbirth, with its nine months warning. Despite this, there is little awareness amongst general psychiatrists of the predictable and manageable risk that faces many of their female patients should they become pregnant, nor of the risks that mentally ill mothers may pose for their children.

This article discusses the likely contribution of childbirth and parity to the excess of affective morbidity in women, the risk posed by childbirth to women who were previously well and to those with pre-existing psychiatric disorder.

\section{Diagnostic status}

One of the reasons why childbirth-related psychiatric disorders attract little attention in general adult psychiatry may be that officially they do not exist. From ICD-7 and DSM-I onwards the special category of postpartum disorder disappeared.

The prevailing view is expressed in ICD-10, which states that "the clinical picture of puerperal psychosis is so rarely if ever reliably distinguishable from affective disorder or schizophrenia that a special category is not justified". This view largely results from earlier studies which showed that both the family history of affective disorder and the subsequent rates of affective illness in women who suffered from puerperal psychosis was similar to those who suffered from such an illness outside of childbirth. Childbirth was therefore seen as a life event, provoking illness in a vulnerable group, and any distinctive clinical characteristics were seen as pathoplastic effects of childbirth. However, Kendell et al (1987) argue that this does not explain the dramatic increase in risk, particularly in the first 90 days following childbirth. Other studies (Kadrmas \& Winokur, 1979; Dean, 1989; Cooper \& Murray, 1995) have suggested that women who have a first episode of severe affective illness following childbirth may have a greater risk of developing a further episode of illness following subsequent childbirths, and a lower order of risk following non-puerperal events.

Future research may reveal that there are two groups of women who become mentally ill following childbirth, those whose mental illnesses are only provoked by childbirth and those for whom childbirth is one of many events which may provoke such an illness. In the meantime it would seem reasonable to define postnatal psychiatric disorder as a new episode of mental illness which arises in temporal relationship to childbirth. It should be classified according to ICD-10 or DSM-IV in a non-postpartum category but should receive an additional classification of a psychiatric disorder associated with childbirth.

Most of the studies referred to in this text, using standardised and operational clinical criteria, place postpartum psychiatric disorder within the spectrum of affective disorders. However, these diagnoses do not do justice to the condition's heterogeneity, lability and complexity, particularly with regard to postpartum psychoses. First-rank

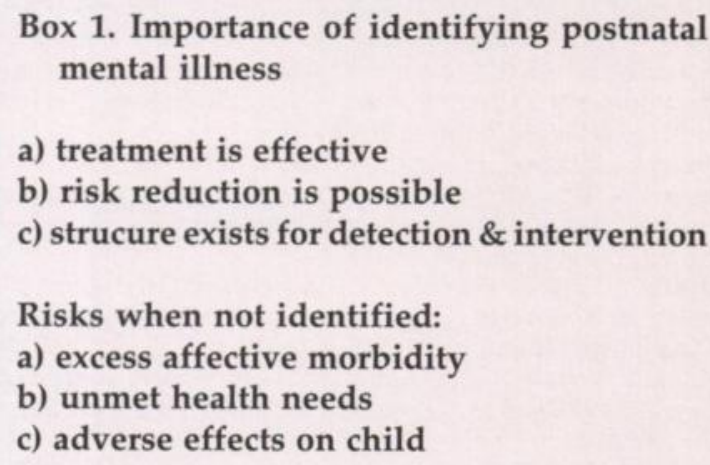


symptoms of schizophrenia are often present (Kadrmas \& Winokur, 1979) and may be sufficient to result in categorisation of the illness as 'schizoaffective'.

\section{Postnatal affective disorder}

\section{Depressive illness}

A substantial proportion of the excess depressive morbidity in young married women is accounted for by childbirth. Ten per cent of women suffer from a postnatal depressive illness (DSM-III-R major depression). Between 3 and $5 \%$ of all women would satisfy the criteria for moderate to severe depressive illness following childbirth, which represents an increase in risk estimated between five and ten-fold (Cox, 1993).

\section{Puerperal psychosis}

It is now well established that two out of every thousand women delivered are admitted to a psychiatric hospital suffering from puerperal psychosis. It is remarkable that the incidence has remained broadly constant for over a century and in different countries (Kendell et al, 1987). It is likely therefore that this incidence will be the same in the future. This figure represents a dramatic increase in risk compared with non-childbearing women (Kendell et al, 1987), but nonetheless admission for puerperal psychosis remains relatively rare. Studies reveal that a further two per thousand women delivered are admitted to hospital suffering from non-psychotic conditions (Kendell et al, 1987; Meltzer \& Kumar, 1985). Not only is the rate of admission to psychiatric hospitals following childbirth increased but also the rate of referral to psychiatric services (Oates, 1988; Kendell et al, 1987). Kendell has estimated the risk of a woman developing a bipolar illness in the first 90 days following delivery as a 16-fold increase over lifetime risk and of being admitted with a psychotic episode as being a 32-fold increase in relative risk. The risk of a woman being referred to a psychiatrist in the year following childbirth is five times greater than at other times in her life (Oates, 1994).

The majority of admissions for psychosis take place within 16 days of delivery and presentations of severe illness within 90 days. Risk is therefore highest in the first three months following delivery.
Childbearing can therefore be seen to pose a major risk to the mental health of women who are previously well. This risk is particularly high in Western societies for first childbirth.

\section{Risk and prenatal psychiatric disorder}

The risk of becoming ill following childbirth posed by a previous history of psychiatric disorder (puerperal or non-puerperal) will be discussed under risk factors. This section considers the risk to the mental health of a woman who is already suffering from a psychiatric disorder, or receiving treatment for it, at the time of conception, i.e. women with chronic or recurrent mental illnesses. This group of illnesses therefore encompasses the entire range of psychiatric conditions that afflict women during their reproductive life span. Apart from depression and anxiety (see review by Kumar, 1985), the prevalence of a given condition in pregnancy is the same as it is in the general agematched female population. Some epidemiological studies (Kendell et al, 1976) even suggest that there may be a slight reduction in the prevalence of mental illness, as judged by contact rates with psychiatric services during pregnancy, including contacts for deliberate self-harm. Obvious exceptions are disorders like anorexia nervosa, which reduce fertility. Schizophrenia has also in the past been associated with reduced fertility (a contribution being made by neuroleptic medication). However, the effects of 'normalisation' of this group of patients, inherent in the move towards community care, may result in an increase of reproductive activity.

Women with chronic or episodic mental illness who become pregnant are the poor relations of those who develop postnatal disorders in terms of

\section{Table 1. Incidence of postnatal affective disorders}

Diagnosis

General practitioner

Major depressive illness

Severe depressive illness

Referred to a psychiatrist

Admitted

Admitted, psychosis
Rates

(\% of women delivered)

15-30 10

3-5

1.7

0.4

0.2 
the amount of research effort and service provision they receive. It is common for a woman who is severely mentally ill to remain as an in-patient on a general adult psychiatric ward until she starts labour, following which her infant is usually cared for by others.

\section{Manic depressive psychosis}

Woman with multiple episodes of bipolar disorder are probably not at increased risk of a deterioration in their state during pregnancy, in contrast to their marked increase in risk of having a puerperal relapse, now estimated as high as one in two (Wieck et al, 1991; Marks \& Wieck, 1992). This risk may be even higher if the woman has been predominantly manic.

\section{Chronic schizophrenia}

Schizophrenic patients do not experience rates of recurrence or exacerbation of their illnesses during pregnancy or following childbirth comparable to those suffering from bipolar disorder (Kendell, 1987). However, this is probably only true if their maintenance neuroleptic medication is continued. The main problem facing these women is the effect of the inevitable stresses and high expressed emotion of childbearing on their mental health and their capacity to meet the needs of their infant (see below).

Although the risk of relapse of a serious chronic or recurrent mental illness is not increased during pregnancy, such a relapse will have major implications for both the developing foetus and the newborn infant. Disturbed behaviour may severely compromise antenatal care and pose a direct and indirect risk to the baby through self-neglect, substance abuse, poor nutrition and hygiene, concealed pregnancies and unassisted delivery. In a small minority of cases, conception may occur whilst a patient is acutely psychotic, because of altered judgement or the ability to consent. In such cases the resultant distress may cause a deterioration in mental health.

Box 2. Risk factors for severe illness

Previous psychiatric history

Previous postnatal illness

Family history of affective disorders

Caesarian section (psychosis only)

\section{Risk factors for postnatal psychiatric illness}

\section{Puerperal psychosis}

The most consistent findings from research are a family history of severe affective disorder and a prior history of psychiatric disorder. These patients are not otherwise distinguishable from the normal obstetric population. Studies from Western countries report first-time (probably emergency) caesarian section as a risk factor for puerperal psychosis. It may be that high levels of obstetric concern during pregnancy and foetal loss are also risk factors. Some studies have reported a trend towards severe affective disorder being more common in women who are rather older and longer married at the time of their first pregnancy and therefore more likely to have had higher education.

Large scale studies have yet to be completed on the effects of new techniques, such as in vitro fertilisation, but the clinical impression from specialist services is that these women are more vulnerable to mental illness than women who conceive spontaneously. Although some risk factors, such as previous severe unipolar or bipolar disorder or family history of severe affective disorder, pose a high degree of risk, approximately $80 \%$ of women with severe affective disorder following their first childbirth have none of these risk factors. The risk factors therefore only account for up to $20 \%$ of the variance of condition. Puerperal psychosis will take most women, their families and their caretakers by surprise.

The prevailing current opinion is that in the causation of puerperal psychoses, biological factors (including genetic factors) are likely to be as important if not more important than psychosocial and obstetric factors. The most plausible explanation for these conditions is that the majority of them are a variant of bipolar disorder and that the women carry this genetically determined susceptibility into childbirth, following which the illness is triggered by some factor. One recent explanation for this 'triggering' mechanism is that such patients develop a hypersensitivity of the central $D_{2}$ receptors, which may be related to the effects of oestrogen withdrawal on the function of the dopamine systems. Other steroid hormones have also been implicated (Wieck, 1989). 


\section{Non-psychotic postnatal depressive illness}

Research findings suggest that psychosocial factors are at least as important, if not more important, than biological factors in the causation of major depressive illnesses in the puerperium. However, there may be a subgroup of patients for whom biological factors are in the ascendancy. They present within the first four weeks after birth with very severe postnatal depressive illnesses, comprising one-third of all referred severe depressive illnesses (Kendell et al, 1987; Oates, 1988).

The risk factors for mild depressive illness, dysthymia and adjustment problems in the puerperium include youth, being single, recent marriage, social adversity, recent life events, psychiatric history, lack of a female confidante, ambivalence about the baby, previous miscarriage or termination of pregnancy. It has been suggested by Elliott (1989) that women who have several of these risk factors suffer from twice as much postnatal depressive illness as those who do not. However, the majority of women who suffer from postnatal depressive illness following their first childbirth will not have been predicted, and the majority of women with identified risk factors will not go on to suffer from postnatal depression. Because these risk factors are relatively nonspecific and widely found in the population they are useful for targeting groups of people for special resources rather than for predicting individuals at risk.

\section{Risk management}

One of the main purposes of identifying risk factors for the development of common and serious illnesses is to be able to reduce risk to future maternal mental health and the developing child. If possible, this primary prevention would be an unusual if not unique event in psychiatry.

\section{Puerperal psychosis and} postpartum relapses of bipolar disorder

Women at high risk of severe affective disorder, and in particular puerperal psychosis, can be identified at the booking clinic by virtue of a prior history of bipolar disorder (postnatal or non-

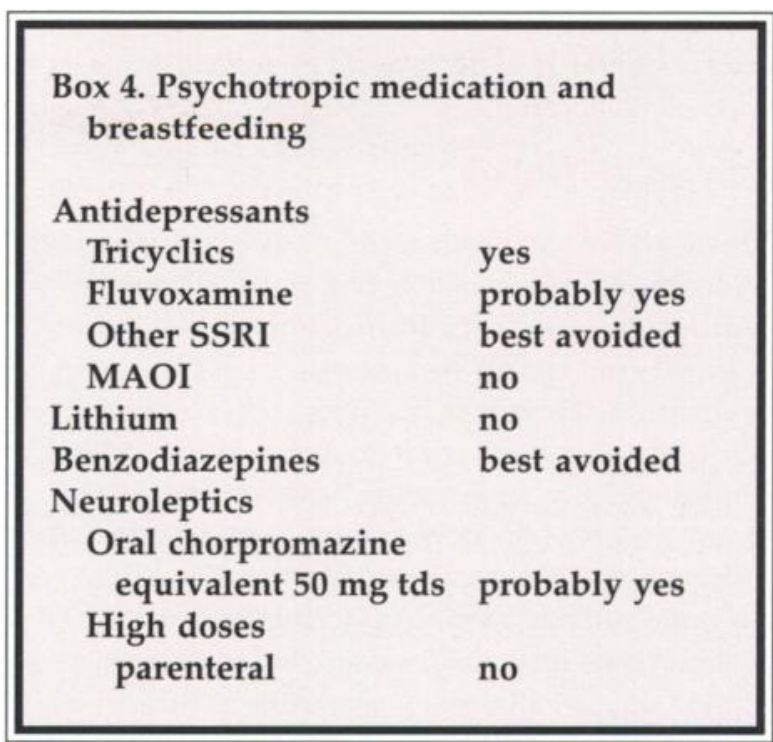

postnatal) or a family history. Such women are at high risk (1:3 or 1:2) of becoming seriously ill in the first 90 days following childbirth, the maximum risk being in the first 16 days for puerperal psychosis.

A woman with a personal history of affective psychosis should be referred during her pregnancy to a psychiatrist with a special interest. This, at the very least, offers the psychiatrist a unique opportunity to know the patient when well and to engage in forward planning. It will also enable those caring for the patient, both professionals and family, to be alert and after delivery to watch for the early signs of the illness and to ensure prompt and appropriate treatment (secondary prevention).

At best it offers the opportunity to engage in prophylactic treatment (primary prevention). Lithium carbonate, started on the first postpartum day, and aiming at a therapeutic level between day 3 and day 5, may prevent the onset of postpartum affective psychoses (Kadrmas \& Winokur, 1979; Stewart et al, 1991). However, this remains to be confirmed by randomised controlled trials. Alternatives, particularly for breast-feeding mothers, include using small doses of neuroleptic medication such as haloperidol or chlorpromazine. This regime should also begin on the first postpartum day. The findings of Wieck et al (1991) suggest a rationale of avoiding the postpartum drop in oestrogen levels. A trial of transdermal oestrogen in the prevention of recurrent postpartum psychosis is under way (Henderson et al, 1991). The use of progesterone, given intramuscularly before birth and rectally for a period of time afterwards, has much popular support (Dalton, 1985). However, neither its scientific basis or clinical effectiveness have been confirmed by others. 


\section{Postnatal depression}

\section{Primary prevention}

For those women with a previous history of early presentation of severe postnatal depressive illness it might appear rational to engage in primary prevention, using hormonal or psychotropic medication. However, there is no evidence from randomised control trials to support this strategy. Nonetheless, women who have suffered from a previous episode of postnatal depression will approach their general practitioners for advice on the possibility of avoiding it after their next baby. In such cases it would be reasonable to start these women on a small dose of a suitable antidepressant (tricyclic if breast-feeding), gradually increasing the dose to therapeutic range. Progesterone prophylaxis (Dalton, 1985) is popular but not of proven efficacy.

In milder cases of postnatal depression it is generally assumed that psychosocial factors play the major role. A study of the effects of psychological intervention (modified antenatal classes) during pregnancy in an identified 'at-risk' population (Elliott, 1989) revealed a reduced rate of postnatal depression at three months, compared with those at-risk women who received standard care. While these findings have yet to be replicated, they suggest that relatively simple intervention might reduce risk.

\section{Secondary prevention}

This is a practical possibility. The postnatal examination carried out on all women in the United Kingdom at six weeks after birth should include either a brief routine clinical examination to detect depressive illness or the use of the Edinburgh Postnatal Depression Scale (Cox \& Holden, 1987). Such a strategy would go a long way towards detecting the $10 \%$ of women likely to be suffering from postnatal depressive illness and would ensure early treatment of this condition, thus reducing avoidable and prolonged psychiatric morbidity and limiting the consequences for the child. Such early treatment for the more severe cases would include antidepressants and, for the less severe cases, nondirective counselling or a cognitive psychotherapeutic approach (Ellliott, 1989; Cooper \& Murray, 1993). A better understanding of the numerous psychosocial risk factors for the development of postnatal depressive illness would enable obstetricians, midwives, health visitors and general practitioners to be more aware of the vulnerable patients that they treat and more alert to the early signs of the illness, as well as to the possibility of reducing certain risk factors, should this be possible.

\section{Pre-existing psychiatric disorder}

One of the most important aspects of risk reduction is an awareness on the part of general adult psychiatrists that women with severe affective disorder or enduring mental health problems might become pregnant. Discussing contraception and the likely effects of pregnancy, not only upon their mental health but also upon their capacity to care adequately for their child physically and emotionally, should be part of ordinary psychiatric practice. Women with bipolar disorder are at particularly high risk of becoming psychotic in the first few weeks following delivery. The lithium that many of them will be receiving to stabilise their mental state is potentially teratogenic. They should be advised to take contraceptives as long as they are taking lithium, to plan their conceptions carefully so that lithium can be withdrawn before they conceive and be restarted immediately following delivery.

\section{Chronic schizophrenia}

The risk for schizophrenic relapse during pregnancy, or in the early months following delivery, is low provided medication is maintained. Although there is no evidence that neuroleptic medication is teratogenic, there is no information about the long-term effects on the developing child of having received such medication in utero or through breast milk. The theoretical risk of dopamine blockade in the developing foetus has to be balanced against the practical risk of a maternal relapse in pregnancy and the puerperium. As there is some evidence (Ruben, 1987) that babies born to mothers on large doses of neuroleptic medication may suffer from both anticholinergic and extrapyramidal side-effects it would seem reasonable to reduce the neuroleptic medication to the lowest possible maintenance dose before delivery, increasing again after delivery.

The most tangible risk to a mother with chronic schizophrenia is the stress and high expressed emotion involved in raising a small child. Clinical impressions from Mother \& Baby Units which offer an assessment programme for mothers with enduring mental health problems suggest that the outcome for mothers suffering from chronic schizophrenia is poor, particularly if they are single and suffer from social adversity. Most of them are 


\section{Box 5. Psychiatric intervention in obstetrics \\ Preconception counselling 'at risk' women \\ Booking clinic screen psychiatric disorder in: - past history - family history \\ Vigilance depression, anxiety in pregnancy, infertility, complications in Caesarian section \\ After delivery \\ 6 week postnatal examination monitor closely screen all mothers for postnatal depression}

unable to care for their children upon leaving the unit, necessitating their removal into care. In order to minimise the risks to both mother and infant it would seem sensible for all general adult psychiatrists managing fertile chronic schizophrenic women to include advice on contraception and pregnancy in their care programme approach, which will include a realistic appraisal of the woman's ability to meet the needs of her child. Her pregnancy should be collaboratively managed by the obstetrician and psychiatrist, with an early involvement of social services so that appropriate plans can be made for her and the infant's care following delivery. One such appropriate plan could be the admission of the mother/infant pair immediately following delivery to a Mother \& Baby Unit. Skilled staff can then undertake an assessment of her ability to care for her infant and of her needs to continue this care in the community. Such an admission would also serve to provide the new mother with an understanding environment in which she can develop the best possible attachment to her child and the skills she needs to care for it (Appleby, 1993).

\section{Reduction of risk to children}

The evidence of the adverse effects on the infant and child's well-being and development broadly comes from two different types of studies with different methodologies. The first follows up the children of large numbers of psychiatrically ill parents, using indirect outcome measures such as health statistics and parent and teachers' assessments. Similar methods have been used for prospective studies of women identified in antenatal clinics, comparing the outcome of children of mothers with postnatal depression with those of well mothers. In general these studies reveal that there is an association between maternal psychiatric disorder and many parameters of childhood morbidity. Their findings support a view that the effects of maternal psychiatric disorder are strongly associated with social adversity and marital disharmony and that chronic disability and personality dysfunction are more important than single episodes of hospitalised major psychiatric disorder (Harder, 1980).

The second kind of study, usually involving smaller numbers, involves the direct assessment of mother/infant interactions and infant and child development using naturalistic observational techniques. Such studies consistently report the adverse effects of maternal depression on mother/ infant attachment, the infant's social and language development and later cognitive development at school. This effect is particularly marked in boys and again appears to be strongest when associated with social adversity and marital conflict (Stein, 1991; Murray, 1992).

\section{Child abuse}

Suicide and infanticide are rare but occasional consequences of puerperal psychosis and profound postnatal depression. Very occasionally a child is killed as the direct result of a delusional state in its parents. Numerically these cases are insignificant when compared to the numerous cases of nonaccident injury in small children in the population as a whole. Few studies have examined the psychiatric status of abusing parents using standardised criteria. There is little evidence to implicate severe mental illness as a major contributor to non-accidental injury in children. The most consistent findings implicate parental youth, prematurity, previous abuse of the parents themselves and in men a previous criminal record for violence. On the other hand, mild depression and anxiety is commonplace in mothers who have abused their children (Oates, 1987).

The most important factors in the reduction of risk to both child and mother from mental illness are prompt detection, early intervention and vigorous treatment of the maternal psychiatric disorder. A lot of attention needs to be paid by those caring for the mother to her relationship with her child. Any specific problems with feeding, sleeping, the need for social support and marital work should be addressed. 


\section{Puerperal psychosis}

Women who require hospitalisation following delivery should be admitted, if possible, to a specialist Mother \& Baby Unit, where the skills to manage the mother/infant relationship and meet the needs of the child will be found.

\section{Postnatal depression}

The less severe illnesses will usually be treated in the community. While for serious depressive illness antidepressant medication will be a critical agent in recovery, for the less severe conditions psychological treatments may be at least as effective as antidepressants (Holden, 1989; Murray, 1994). Counselling using a cognitive-behavioural approach may be helpful for mother/infant relationships. For all types of psychiatric disorder where physical contact is encouraged between mother and child, verbal and social interaction and breastfeeding can help minimise the possibility of adverse sequelae. Although there is probably not an increased risk of child abuse in women with severe postnatal psychiatric disorder, complaints of irritability towards children and the expression by the mother of fears that she might harm her child should always be taken seriously, and discussions with the patient about her ability to care for her children and her feelings towards them should be part of every assessment.

\section{References}

Appleby, L. \& Dickens, C. (1993) Mothering skills of women with mental illness. British Medical Journal, 306, 348-349.

Cooper, P. \& Murray, L. (1995) The course and recurrence of postnatal depression. British Journal of Psychiatry, 166, 191195.

Cox, J. L., Holden, J. M. \& Sagovsky, R. (1987) Detection of postnatal depression: development of the 10-item Edinburgh postnatal depression scale (EPDS). British Journal of Psychiatry, 150, 782-786.

-, Murray, D. \& Chapman, G. (1993) A controlled study of the onset prevalence and duration of postnatal depression. British Journal of Psychiatry, 163, 27-31.

Dalton, K. (1985) Progesterone prophylaxis used successfully in postnatal depression. Practitioner, 229, 507-508.

Dean, C., Williams, R. J. \& Brockington, I. F. (1989) Is puerperal psychosis the same as bipolar manic-depressive disorder? A family study. Psychological Medicine, 19, 637-647.

Elliott, S. A. (1989) Psychological strategies in the prevention and treatment of postnatal depression. Baillière's Clinical Obstetrics \& Gynaecology, 3, 879-904.

Harder, D. W. (1980) Child competence and psychiatric risk IV relationships of parent, diagnostic classification and parent psychopathology: severity to child functioning. Journal of
Nervous and Mental Disease, 1866, 343-347.

Henderson, A., Gregoire, A. \& Kumar, R. C. (1991) Treatment of severe postnatal depression with oestradiol skin patches. Lancet, 338, 816-817.

Holden, J. M., Sagovsky, R. \& Cox, J. L. (1989) Counselling in a general practice setting: a controlled study of health visitors; intervention in treatment of postnatal depression. British Medical Journal, 298, 223-226.

Kadrmas, A. \& Winokur, G. (1979) Post-partum mania. British Journal of Psychiatry, 135, 551-554.

Kendell, R. E., et al (1976) The influence of childbirth on psychiatric morbidity. Psychological Medicine, 6, 297-307.

-, Chalmers, L., Platz, C. (1987) The epidemiology of puerperal psychoses. British Journal of Psychiatry, 150, 662673.

- (1985) Pregnancy, childbirth and mental illness. In Progress in Obstetrics and Gynaecology, vol. 5 (ed J. Studd) pp. 146-159. Edinburgh: Churchill Livingstone.

Marks, M. N., et al (1992) Contribution of psychological and social factors to psychotic and non-psychotic relapse after childbirth in women with previous histories of affective disorder. Journal of Affective Disorder, 29, 253-264.

Meltzer, E. S. \& Kumar, R. (1985) Puerperal mental illness, clinical features and classification: a study of 142 mother and baby admissions. British Journal of Psychiatry, 147, 647-654.

Murray, L. (1992) The impact of postnatal depression on infant development. Journal of Child Psychology and Psychiatry, 33, 543-562.

— \& Cooper, P. (1994) Three psychological treatments for postpartum depression: a comparative trial of impact on mothers and their infants. Marcé Society International Conference Proceedings, Cambridge.

Oates, M. R. (1987) Different types of abusing parents. In Understanding Child Abuse, 2nd edn (ed D. Jones). London: Hodder \& Stoughton.

- (1988) The development of an integrated communityorientated service for severe postnatal mental illness. In Motherhood and Mental Illness, Vol. 2 (eds R. Kumar \& I. F. Brockington). London: Wright.

- (1994) Postnatal mental illness: organisation and function of a psychiatric service. In Aspects of Perinatal Psychiatry: use and misuse of the EPDS (eds J. Cox. \& J. Holden). London: Gaskell.

Ruben, P. C. (1987) Prescribing in Pregnancy. London: British Medical Association.

Stein, A., Gath, D. H., Bucher, J., et al (1991) The relationship between postnatal depression and mother-child interaction. British Journal of Psychiatry, 158, 46-52.

Stewart, D. E., Klompenhouwer, J. L., Kendell, R. E., et al (1991) Prophylactic lithium in puerperal psychosis: The experience of three centres. British Journal of Psychiatry, 158, 393-397.

Wieck, A. (1989) Endocrine aspects of postnatal mental disorders. Baillières Clinical Obstetrics \& Gynaecology, 3, 857877.

—, Kumar, R., Hirst, A. D., et al (1991) Increased sensitivity of dopamine receptors and recurrence of affective psychosis after childbirth. British Medical Journal, 303, 613-616.

\section{Multiple choice questions}

1 The following drugs can be given to breast feeding mothers:

a Benzodiazepines

b Lithium carbonate

c Sertraline

d Dothiepin

e Clozapine 
2 Risk factors for puerperal psychosis include:

a Foreign born

b Age under 21

c Family history of bipolar disorder

d Previous episode mania

e Chronic schizophrenia

3. Women following childbirth are at increased risk of:

a Suicide

b Alcoholism

c Deliberate self harm

d Bipolar illness

e Child abuse

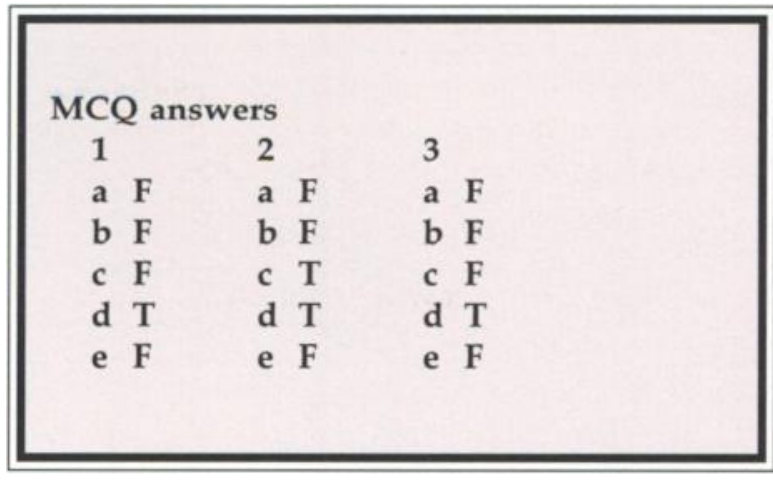

\section{Correspondence}

\section{Chronic fatigue syndrome}

SIR: The chronic fatigue syndrome article by Lynch (APT, November 1994, 1, 33-40) mentions antidepressants can be used in this condition, but made no specific mention of the 'psychic energisers' which have been available for many years. These are the old MAOIs which are well known to overcome fatigue as well as reduce anxiety and depressive symptoms. Unfortunately they are classed as antidepressants and their adverse effects over-emphasised. Isocarboxazid is particularly safe as long as the MAOI diet is adhered to - it is also cheap.

This paper confirmed to me that many psychiatrists no longer acquire the skill of prescribing the 'old' MAOIs. This is obvious as a MRCPsych Part II examiner, and it means the chronic fatigue syndrome is often not treated as vigorously as it should be.

Severn NHS Trust

B. H. ANSTEE

Horton Road

Gloucester GL1 3PX

\section{Electroconvulsive therapy}

SIR: I am concerned about some of the MCQ answers in Lock's article on electroconvulsive therapy (APT, November 1994, 1, 47-56), specifically with regard to the Mental Health Act. Indeed the advice in the text is also less than clear.
MCQ 4a - Patients are entitled to receive treatment without a clear explanation if they wish so to do, and their consent to the lack of information is in itself valid.

MCQ $4 b$ - It may occasionally be appropriate to give patients ECT against their wish following their changing their minds with regard to consent, if the patient's condition warrants this. This would either be under the consultant's duty of care to a patient who was informal, or under Section 62, subsection 2, for patients who are detained under an appropriate Section of the Mental Health Act.

MCQ 4c - Detained patients are entitled to give consent to ECT. It requires the completion of Form 38 by the RMO.

It is worth emphasising that Section 62, subsection 2, states "Sections 60 and 61 shall not preclude the continuation of any treatment, or of treatment under any plan pending compliance with Section 57 or 58 above, if the discontinuance of the treatment, or of treatment under the plan would cause serious suffering to the patient".

Having made these criticisms, may I thank you for your work in producing a journal which looks as if it will make continuing professional development a pleasure, rather than a chore.

St. Mary's Hospital

A. S. ZiGMOND

Leeds LS12 3QE 